\title{
Silencing Akt1 Enhances the Resistance of Prostate Cancer Cells to Starvation and Inhibits Starvation- Induced Lung Metastasis Through Epithelial- Mesenchymal Transition in Prostate Cancer
}

\section{Mei Yang}

Chongqing Medical University

Hui Liu

Chongqing Medical University

Fei Gao ( $\square$ gaofei@hospital.cqmu.edu.cn )

Chongqing Medical University https://orcid.org/0000-0001-6973-2013

Guo ping Qiu

Chongqing Medical University

\section{Research Article}

Keywords: Nutritional starvation, slow-growing cells, Silencing Akt1, lung metastasis

Posted Date: October 25th, 2021

DOl: https://doi.org/10.21203/rs.3.rs-898725/v1

License: (c) (i) This work is licensed under a Creative Commons Attribution 4.0 International License.

Read Full License

Version of Record: A version of this preprint was published at Medical Oncology on November 10th, 2021. See the published version at https://doi.org/10.1007/s12032-021-01600-z. 


\section{Abstract \\ Background}

Nutritional starvation (NST) is the basis of tumor anti-angiogenesis and metabolic therapy strategy. Silencing Akt1 inhibits prostate cancer ( $\mathrm{PCa}$ ) cells growing; slow-growing cells tend to consume less nutrition. It is suggested that Akt1 silenced cancer cells will have a more substantial tolerance to NST. Clarify this critical question is vital for tumor treatment strategies based on Akt1 and NST.

\section{Methods}

The Akt1 gene of PC3 and DU145 cells was silenced by lent-virus. NST model was established by serum stripping. Cell viability was detected by MTT assay and cell counting method; Apoptosis was detected by TUNEL and flow cytometry, and cell invasion was determined by transwells and ECIS. The markers of Epithelial-mesenchymal transition (EMT) were detected by western Blotting. PCa lung metastasis model was established by tail vein injection and quantified by Indian ink and GFP fluorescence.

\section{Results}

Silencing Akt1 slowed down the decrease of cell number and increase of apoptosis caused by NST. Silencing Akt1 with NST exposure in PCa cells could down-regulate epithelial markers (E-cadherin, claudin-5, and ZO-1) and up-regulate mesenchymal markers N-cadherin and EMT regulators Snail. Although silencing Akt1 enhanced the invasion of PCa cells induced by NST in vitro, silencing Akt1 inhibited the PCa lung metastasis induced by NST in vivo.

\section{Conclusions}

Silencing Akt1 gene enhances the resistance of PCa cells to NST. The invasion results in vitro were inconsistent with those metastases in vivo, which may be related to a combination of NST with silencing Akt1 to maintain the mesenchymal state of PCa cells through EMT.

\section{Introduction}

Prostate cancer ( $\mathrm{PCa}$ ) is the most common cancer in men; it is the second leading cancer-related death among men in the United States [1]. In the process of tumor proliferation, nutrients are required for solid tumors to grow. Without nutrition support, tumors cannot grow to a particular size. This phenomenon is also known as the Warburg effect [2]. Many therapeutic strategies aimed to cut off the fuel supply in cancer cells, such as anti-angiogenesis and metabolism strategy, which have achieved a good beginning and once brought hope, but finally, scientists found that starvation could not kill all cancer cells and halt cancer's progress. 
On the one hand, starvation is cellular stress that induces apoptosis or death of cancer cells[3]; conversely, cancer cells adapt to harsh environments through a series of changes. Through these processes, the invasive ability of cancer cells is enhanced[4]. After fasting exposure, cancer cells obtain the ability of invasion and metastasis through the Epithelial-mesenchymal transition (EMT) process [5]. The previous study demonstrated that EMT process enhanced the invasion and metastatic ability of cancer cells[6], acquired stem cell-like characteristics, so as to reduce proliferation and demand for nutrients $[7,8]$. These changes might reduce nutrition consumption and facility cancer cells to survive in starvation.

Our previous study found that silencing Akt1 could inhibit the growth and proliferation of PCa cells[9] and enhance cancer cell invasion via EMT[10], suggesting that silencing Akt1 might reduce nutrition consumption in PCa cells. Using bioinformatics analysis, we found that EMT has some relationship with lipid and glucose metabolism by analyzing EMT-related molecules (Supplementary Fig. 1A). Further cluster analysis confirmed that Akt1 was related to several starvation-related pathways such as oxidative stress-induced cell death and glucose metabolic process et al. (Supplementary Fig. 1B). These results implied Akt1 silencing might have the potential ability to enhance the starvation resistance of cancer cells.

Akt1 is an important regulator of cancer cell metastasis and invasion. Our previous studies have confirmed that silencing Akt1 significantly enhanced the metastasis of PCa [10]. The invasion in vitro and metastasis ability of cancer cells in vivo may be inconsistent, because they are essentially two different processes. The metastasis at the animal level is much more complex than the invasion at the cell level, and there are more influencing factors [11]. The present study found that Akt1 silencing enhanced invasion ability in vitro, however, it unexpectedly inhibited lung metastasis induced by starvation of PCa cells in vivo. This phenomenon that the results of in vivo experiments are inconsistent with those of in vitro experiments once again confirms the difference in invasiveness and metastasis.

Our study found the role of Akt1 in the starvation of PCa. Silencing Akt1 enhances the resistance of PCa cells to starvation. Maintaining the mesenchymal state of PCa cells through EMT could enhance the invasion ability of cancer cells in vitro; however, it inhibited the metastasis of PCa cells in vivo.

\section{Materials And Methods}

\section{Cell culture and Animals}

Human PCa cell lines DU145 and PC3 were purchased from ATCC (Manassas, VA). HUVECs were obtained from Lonza (CC-2527; Lonza, Allendale, NJ). Cells were maintained as previously described[12].

Eight-week-old male nude mice (22-26g) were obtained from the animal experiment management center of Chongqing Medical University (CQMU) (BALB/c background, Chongqing, China). The ethics board of CQMU approved this study. All animal experiments were carried out following guidelines set by CQMU. 


\section{Bioinformatics analysis of regulatory molecules of EMT}

44 EMT-related molecules were collected by manual search combined with our knowledge background (the list of the genes is available in supplement table1). Cytoscape 3.8.0 was used to analyze the function enrichment of EMT-related genes including the Akt1 gene [13, 14].

\section{Generated ShAkt1 stable cell lines}

PC3 and DU145 cells with stable knock-down of Akt1囚ShRNA Akt1 deficient, Sh Akt1区and respective Control group (Control) were generated usinghU6-MCS-EGFP lentiviral vector ( $10^{9}$ p.f.u.) (JiKai, Shanghai China). The stable ShAkt1 cell line was developed as previous study [10].

\section{Cell viability assay}

DU145 and PC3 cells were plated at a density of 1000 cells/well in a 96-well plate with 100 ul growth medium at each well. At the indicated time-point, treated cells were washed and resuspended in PBS. Then, fresh media containing $20 \mu \mathrm{l}$ of MTT (5 mg/ml stock) was added and incubated for another 4 hours. Results were measured by taking absorbance at $540 \mathrm{~nm}$. Four times independent experiments were performed.

\section{Foci (colony) formation assay}

The cells were seeded into 6-well plates at a concentration of 500 cells per well. After 14 days of standardized culture, colony counts and rates were calculated after the colonies were fixed with absolute methanol and stained with $0.1 \%$ crystal violet. Colonies of $>50$ cells were counted.

\section{Detection of apoptosis by flow cytometry}

According to the manufacturer's instructions, the cells were simultaneously stained with Alexa Fluor 488conjugated Annexin-V and PI, using the Vybrant Apoptosis Assay kit (Molecular Probes, USA). Samples were analyzed by Facscalibur flow cytometer. The percentage of apoptotic cells was estimated utilizing the Cell Quest Pro software. The simultaneous staining of cells with Annexin-V and PI allowed the resolution of viable cells (A-/PI-), early apoptotic cells (A+/PI-), necrotic cells (A-/PI+), and late apoptotic cells $(\mathrm{A}+/ \mathrm{PI}+)$.

\section{Cancer cells invasion detected by Transwells}

Invasion assay was performed using matrigel invasion chambers were carried out as the previous study[12]. Briefly, DU145 and PC3 cells were seeded on the top chambers; after 10 hours of incubation, the upper chamber cells were wiped off with a cotton swab. The invaded cells in the lower membrane surface were fixed with eosin staining. The cells were counted under a microscope (Olympus, Japan).

\section{ECIS assay for cancer cell micro-invasion}


The ability of cancer cells to penetrate the endothelial cell monolayer was used to detect the migration (micro invasion) of cancer cells across the endothelial cell monolayer, which was measured by electric cell-substrate impedance sensing (ECIS) technology as previously described(Applied Biophysics, Troy, NY) [15].

\section{Terminal deoxynucleotidyl transferase-mediated dUTP nick end labeling (TUNEL) assay}

The TUNEL assay for the in situ detection of apoptosis was performed using an apoptosis detection kit (Roche, California, USA) following the manufacturer's instructions. Briefly, cells were first fixed with $4 \%$ paraformaldehyde for 30 minutes at room temperature and then permeabilized with $0.1 \%$ Triton X-100. The cells were then fixed in $20 \mu \mathrm{g} / \mathrm{ml}$ protease $\mathrm{K}$ for 20 minutes, after that, stained with a TUNEL reaction mixture. TUNEL positive cells are brown in the nucleus.

\section{Lung metastasis analysis in vivo}

Nude mice were randomly divided into four groups. DU145 cells $\left(0.5 \times 10^{6}\right)$ suspended in sterile normal saline were injected via the tail vein (i.v.). The metastasis tumor nodules were stained white for the twoweek mouse model as in the previous study[12]. For three days mouse model, mice lung tissue was made into frozen sections. The number of GFP-containing cancer cells was calculated under a confocal imaging microscope (LSM510, Carl Zeiss, Germany), as described previously[10].

\section{Western blot analysis}

Western blotting was performed as described previously[16]. Briefly, the PVDF membrane was incubated with primary antibodies, Caspase-3, E-cadherin, ZO-1, Claudin-5, N-cadherin (1: 1000 dilution, CST, Massachusetts, USA), and $\beta$-actin (1: 5000 dilution, Abcam, Cambridge, MA), respectively. After that, the membrane was incubated with secondary antibodies (1: 5000, Abcam, Cambridge, MA). After developing, the protein expression was observed.

\section{Statistical Analysis}

All the data were expressed as Mean + SD and calculated from multiple independent experiments. The Student's two-tailed t-test or ANOVA was used to determine significant differences among groups using SPSS 11.0 software. Data with $p<0.05$ were considered to have significant differences.

\section{Results}

\section{Silencing Akt1 gene enhanced the resistance of prostate cancer cells to starvation.}

To explore PCa cells' response to nutrient starvation (NST). We developed ten cycles of NST of PCa cells to establish a cell starvation model to mimic the clinic environment, in which PCa cells were exposed to NST repeatedly. 
To assay the function of Akt1 gene on NST of PCa cells, we establish PCa cells with stable Akt1 silencing. We used MTT and Cell number methods to detect cell growth. Our study found that the number of cells and OD value in the Sh Akt1 + NST group decreased significantly compared with the Control + NST group (Fig.1 A-D, p<0.01).

Then, we used colony formation to study cell proliferation after starvation. We found that silencing Akt1 gene inhibited cell proliferation in NST significantly and the colony number of Sh Akt1 + NST group decreased significantly compared with the Control + NST group (Fig.1 E-H, p<0.05).

\section{Silencing Akt1 gene significantly inhibited starvation-induced apoptosis of PCa cells.}

In this study, TUNNEL staining and flow cytometry were used to evaluate the apoptosis of each group. Our study found that starvation significantly upregulated the number of apoptotic cells in the

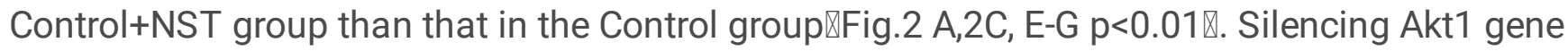
significantly inhibited starvation-induced apoptosis of PCa cells. The number of apoptosis cells decreased significantly compared with the Control + NST group detected by TUNNEL and flow cytometry in the Sh Akt1 + NST groupهFig.2 A,2C, E-G p<0.05区.

Because the primary purpose of the present study is not to explore the apoptotic mechanism of PCa cells in starvation, we only detected that the level of Caspase-3, which is the primary executor of apoptosis[17]. We found that Caspase- 3 in the Control+NST group was the highest among the all four groups $\mathbb{R}$ Fig.2B, 2D $p<0.01 \rrbracket$. Starvation could up-regulate the expression of Caspase-3 in Control group, while starvation could not up-regulate Caspase-3 in ShAkt1 cells. There was no significant difference in the level of Caspase-3 between Sh Akt1 group and Sh Akt1 + NST group 『Fig.2 B, 2D p<0.01区.

\section{Silencing Akt1 gene enhanced PCa cell invasion induced by starvation in vitro.}

The transwells method was used to detect invasion ability of cancer cells. We found that the invasive cell

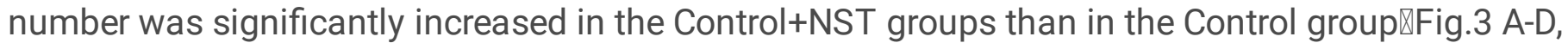
$p<0.01 \rrbracket$. Silencing Akt1 gene further enhanced PCa cells' invasion induced by NST, which manifested by

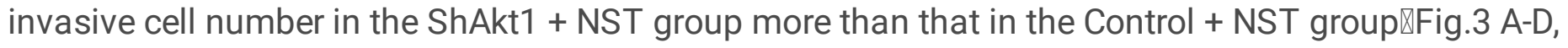
$\mathrm{p}<0.01$.

To verify the results of transwells, we used ECIS to detect the invasion of PCa cells. The data showed PCa cells penetrated vascular endothelial cells, leading to the destruction of the vascular endothelial cell barrier, resulting in the decline of their resistance [18]. Just as transwells data, ShAkt1 + NST group cells had the most substantial reduction in electrical impedance among all the groups detected by ECIS $\otimes$ Fig. 3 $E-F, p<0.01 \rrbracket$.

\section{Silencing Akt1 gene inhibited lung metastasis induced by starvation in vivo.}

To investigate whether starvation increased lung metastasis of PCa cells in vivo, we generated two types of animal metastasis models. One model harvested lung three days after PCa cells injection. The other 
model harvested lung two weeks after PCa cells injection.

In two-week models, we found that NST or Akt1 silencing alone significantly enhanced lung metastasis and the tumor nodules of the Sh Akt1 group and Control+NST group increased significantly compared with Control group $\otimes F i g .3 \mathrm{G}, 3 \mathrm{I}, \mathrm{p}<0.01 \rrbracket$. Surprisingly, if the PCa cells stabled with silencing Akt1 gene were combined with starvation, the ability to induce lung metastasis became severely weakened and the tumor nodules of the Sh Akt1+NST group decreased significantly than that of Sh Akt1 and Control+NST group QFig. $3 \mathrm{G}, 3 \mathrm{l}, \mathrm{p}<0.01 \rrbracket$. To explore whether these results were caused by cell growth or the proliferation of cancer cells in lung tissues, we detected GFP-positive PCa cells by fluorescence in three-day models. We got similar results as the two-week model, that is , GFP-positive cancer cells of the ShAkt1 + NST group decreased significantly than that of Sh Akt1 and Control+NST group冈Fig. $3 \mathrm{H}$ and 3J, $p<0.01 \rrbracket$.

\section{The combination of NST and silencing Akt1 maintained the mesenchymal state of PCa cells.}

E-Cadherin, ZO-1, and Claudin-5 are epithelial cell marker molecules, and N-Cadherin is a typical mesenchymal cell marker molecule. We detected the levels of these molecules by western blotting.

As our previous study [10], we found silencing Akt1 initiated EMT of PCa cells. Compared with the Control group, N-Cadherin increased significantly, while E-Cadherin and Claudin-5 decreased significantly in the Sh Akt1 group. Other epithelial marker molecules, ZO-1, the levels of each group were very low and WB was difficult to detect in PC3 cells, but the trend was the same as that of Claudin-5 in DU145 (Fig.4A-B, C$G, p<0.01)$.

PCa cells treated combined with starvation and silencing Akt1 were found to maintain the mesenchymal state. Compared with the Control group, the level of N-Cadherin was significantly increased, while ECadherin and Claudin-5 decreased significantly in the Sh Akt1 + NST group. And the N-Cadherin of Sh Akt1 + NST was increased than that of Sh Akt1 group (Fig.4 A-B, C-G, $p<0.01$ ).

Snail is an essential regulator of EMT; the up-regulation of Snail was reported to initiate EMT [19]. We found that the Snail of Sh Akt1group was significantly higher than that of Control group. And the Snail of Sh Akt1 + NST was higher than that of Sh Akt1 group, which is the highest among the four groups (Fig.4 $A-B, H, p<0.01)$.

\section{Discussion}

Nutrient starvation (NST) is the basis for anti-angiogenesis and cancer metabolism strategy[20]. However, research has confirmed that NST could not cure cancer. On the contrary, starvation makes cancer cells adapt to adverse conditions, and the surviving cells become more aggressive via EMT[5, 21]. Our study also confirmed that NST or silencing Akt1 promoted the EMT process of PCa cells. Interestingly, we found Akt1 silencing enhanced invasion ability induced by starvation of PCa cells in vitro; however, it inhibited starvation-induced lung metastasis of PCa cells in vivo unexpectedly. These results facility our understanding of PCa metastasis and have a significant clinical and scientific value. 
The literature and our previous study reported that starvation or silencing Akt 1 could enhance cancer cell invasion ability $[4,10,22]$. Till now, little is known about the function of Akt1 on starvation cells. In this study, the bioinformatics method was used to predict the potential relationship between Akt1 and starvation. 44 EMT-related molecules (including Akt1) were collected by manual search combined with our knowledge background (the list of the genes is available in supplement table1). We found that EMT is related to lipid and glucose metabolism by analyzing EMT-related molecules (Supplementary Fig. 1A). Further cluster analysis confirmed that Akt1 was related to several starvation-related path ways such as oxidative stress-induced cell death and glucose metabolic process and so on (Supplementary Fig. 1B).

Previous studies demonstrated starvation slows down the growth of cancer cells [23, 24]. To explore the role of Akt1 in this process, we compared the responses of Control and ShAkt1 cells in NST. We found that the number of cells, OD value and colony number in the ShAkt1 + NST group were decreased significantly slowly compared with those in the Control + NST group (Fig. 1A-H). These results suggested silencing Akt1 gene enhanced the resistance of PCa cells to starvation. Intervening Akt1 may be not good for anti-angiogenesis and cancer metabolism strategy therapies, because these two therapies involve the process of starving cells.

PCa cells undergo apoptosis after serum starvation [25]. Our study also found, compared with control group, apoptosis of Control+NST was increased significantly. However, after silencing Akt1 gene, apoptosis in Sh Akt1 + NST group was significantly inhibited compared with Control + NST group in the same starvation environment. These results suggest that silencing Akt1 gene can significantly inhibit the apoptosis of PCa cells induced by starvation. Further, we detected the expression of Caspase-3, the executor of apoptosis. The result showed that the Caspase-3 levels of Control group were increased after NST. However, starvation could not up-regulate Caspase-3 of ShAkt1 PCa cells, there was no significant difference in the level of Caspase-3 between Sh Akt1 group and Sh Akt1 + NST group(Fig. 2B, 2D). This may be one of the mechanisms for Sh Akt1 cells to resist apoptosis in starvation. At the same time, it also suggested that Caspase- 3 could be regulated by Akt1.

In this study, we found that the results were similar to those in vitro, silencing Akt1 gene or NST alone significantly enhanced cancer cells' ability to induce lung metastasis (Fig. 3). Interestingly, the combination of starvation with silencing Akt1 significantly inhibited the lung metastasis of PCa cells in vivo, but significantly increased the invasiveness of PCa cells in vitro.(Fig. 3). This interesting result may be related to the following reasons. Although the enhancement of invasion ability of cancer cells in vitro generally indicates that their metastasis was also enhanced in vivo, the invasion ability in vitro and metastasis in vivo are essentially different in fact. In particular circumstances, there might be inconsistencies for invasion and metastasis of cancer cells between in vivo and in vitro data [11].

EMT is an important mechanism regulating the invasiveness and metastasis of cancer cells, it is a dynamic and reversible process, and cancer cells need to switch between epithelial or mesenchymal phenotypes. The ability of cancer cells to transform between epithelium and mesenchymal is called EMT plasticity $[26,27]$. Excessive maintenance of cancer cells in a certain epithelial or mesenchymal state 
may be detrimental to cancer cell metastasis $[11,28]$. The present study found that, Starvation or silencing Akt1 alone initiates the EMT process of PCa cells, and the expression of epithelial markers were down-regulated while the levels of mesenchymal markers were up-regulated(Fig. 4). In the combination of starvation and silencing Akt1, the mesenchymal characteristics of Sh Akt1 + NST cells were more obvious. Moreover, Snail, an important EMT-TFs molecule, its level of Sh Akt1 + NST cells was the highest among all groups (Fig. 4). Recent studies have found that overexpression of Twist1, an EMT-TFs molecule, could maintain the cancer cells in a mesenchymal state, and the ability of cancer cells to distant metastasis will be significantly inhibited[11,28]. Based on the above research results, we speculate that Snail expression induced by the combination of starvation and silencing Akt1, might maintain the mesenchymal phenotype of PCa cells, which is not conducive to lung metastasis. This may be the reason for the inconsistent results of the present study in vivo and in vitro.

E-Cadherin is an epithelial marker and considered as an important EMT marker of cancer cells. The decrease in E-Cadherin level indicates EMT initiation [29]. Because the invasion ability of cancer cell is enhanced via EMT, most scholars thought that a decrease in E-cadherin's level could facilitate the metastasis of cancer cells. Actually, E-Cadherin's role in the metastasis of cancer cells is controversial. Some studies have confirmed that metastasis of cancer cells requires involvement of this molecule [30].On the contrary, one study reported that the metastasis of cancer cells is independent of E-Cadherin [31].

We used DU145 cells, which E-Cadherin level is very low, to establish a lung metastasis model. The ECadherin of Sh Akt1 cells is too low to detect by WB. We found that the lung metastasis caused by Sh Akt1 cells was stronger than that in the Control cells, which with relatively high E-Cadherin. If these results suggest that low E-Cadherin is conducive to metastasis, it is difficult to explain why the Control + NST cells caused more lung metastasis than that of Control cells with higher levels of E-Cadherin. This phenomenon that whether up-regulate or down-regulate E-Cadherin could lead to the increase of metastasis, suggesting that E-Cadherin may not play a major role in the process of PCa metastasis in the present study. The difference in metastatic ability in this study may be related to silencing Akt1 gene and starvation. Of course, the specific role of E-Cadherin in PCa metastasis needs to be further studied.

In summary, our study found Akt1 or NST could initiate the EMT, enhance PCa cells' invasion ability, and promote the apoptosis of PCa cells through Caspase-3 in vitro. PCa cells treated with silencing of Akt1 gene and NST may maintain the phenotype of mesenchymal cells, inhibit PCa cells' metastasis in vivo (Supplementary Fig. 2). These results are helpful for the treatment of PCa based on NST theory, such as anti-angiogenesis or cancer metabolism strategy. Further study is required to elucidate this inconsistency's mechanism in vitro and in vivo and clarify E-cadherin's role in cancer cell metastasis.

\section{Declarations}

\section{Ethical approval and consent to participate}


This study was approved by the Ethics Committee of Chongqing Medical University (Chongqing, China). All animal experiments were performed following animal protocols approved by the Chongqing Medical University, following the guideline and regulations for Animal Health and Use (National Standardization Administration of China, 2016).

\section{Consent for publication}

Not applicable.

\section{Availability of data and materials}

The datasets used and analyzed during the current study are available from the corresponding author on reasonable request.

\section{Competing interests}

The authors declare that they have no competing interests.

\section{Funding}

Funds were provided by the National Nature Science Foundation of China to Fei Gao (No.81672893) and Mei Yang (NO.81971230 and 81671312). Furthermore, it is supported by Chongqing Science and health joint project $\ 2020$ GDRC007囚. This work has been accomplished using the resources of the Senior Young-

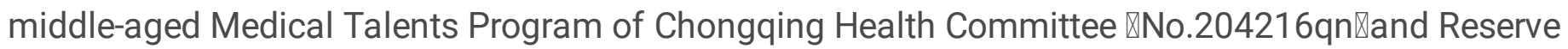
Talents Program for academic Leaders of the First Affiliated Hospital of Chongqing Medical University $\llbracket$ No.XKTS070 to Fei Gao. Science and technology research project of Chongqing Education Commission (KJQN201800428) to Mei Yang.

\section{Authors' contributions}

Conception and design: Fei Gao; Data production, Analysis, and interpretation: Fei Gao, Mei Yang, Hui Liu, and Guo Ping Qiu.; writing the manuscript: Fei Gao and Mei Yang. All authors reviewed the manuscript and accepted the content.

\section{Acknowledgments}

This work has been accomplished using the resources and facilities at Chongqing Medical University.

\section{References}

1. Benke IN, Leitzmann MF, Behrens G, Schmid D. Physical activity in relation to risk of prostate cancer: a systematic review and meta-analysis. Annals of oncology : official journal of the European Society for Medical Oncology. 2018;29:1154-79. 
2. Vander Heiden MG, Cantley LC, Thompson CB. Understanding the Warburg effect: the metabolic requirements of cell proliferation. Science. 2009;324:1029-33.

3. Marchesi F, Monti P, Leone BE, Zerbi A, Vecchi A, Piemonti L, et al. Increased survival, proliferation, and migration in metastatic human pancreatic tumor cells expressing functional CXCR4. Cancer research. 2004;64:8420-7.

4. Garcia-Jimenez C, Goding CR. Starvation and Pseudo-Starvation as Drivers of Cancer Metastasis through Translation Reprogramming. Cell metabolism. 2019;29:254-67.

5. Zada S, Hwang JS, Ahmed M, Lai TH, Pham TM, Kim DR. Control of the Epithelial-to-Mesenchymal Transition and Cancer Metastasis by Autophagy-Dependent SNAI1 Degradation. Cells. 2019;8.

6. Mittal V. Epithelial Mesenchymal Transition in Tumor Metastasis. Annual review of pathology. 2018;13:395-412.

7. Sakunrangsit N, Ketchart W. Plumbagin inhibits cancer stem-like cells, angiogenesis and suppresses cell proliferation and invasion by targeting Wnt/beta-catenin pathway in endocrine resistant breast cancer. Pharmacological research. 2019:104517.

8. Aigner K, Dampier B, Descovich L, Mikula M, Sultan A, Schreiber M, et al. The transcription factor ZEB1 (deltaEF1) promotes tumour cell dedifferentiation by repressing master regulators of epithelial polarity. Oncogene. 2007;26:6979-88.

9. Wang Q, Chen X, Hay N. Akt as a target for cancer therapy: more is not always better (lessons from studies in mice). Br J Cancer. 2017;117:159-63.

10. Gao F, Alwhaibi A, Sabbineni H, Verma A, Eldahshan W, Somanath PR. Suppression of Akt1-betacatenin pathway in advanced prostate cancer promotes TGFbeta1-mediated epithelial to mesenchymal transition and metastasis. Cancer letters. 2017;402:177-89.

11. Ocana OH, Corcoles R, Fabra A, Moreno-Bueno G, Acloque H, Vega S, et al. Metastatic colonization requires the repression of the epithelial-mesenchymal transition inducer Prrx1. Cancer cell. 2012;22:709-24.

12. Gao F, Alwhaibi A, Artham S, Verma A, Somanath PR. Endothelial Akt1 loss promotes prostate cancer metastasis via beta-catenin-regulated tight-junction protein turnover. Brit J Cancer. 2018;118:146475.

13. Reimand J, Isserlin R, Voisin V, Kucera M, Tannus-Lopes C, Rostamianfar A, et al. Pathway enrichment analysis and visualization of omics data using g:Profiler, GSEA, Cytoscape and EnrichmentMap. Nat Protoc. 2019;14:482-517.

14. Rajput A, Thakur A, Sharma S, Kumar M. aBiofilm: a resource of anti-biofilm agents and their potential implications in targeting antibiotic drug resistance. Nucleic Acids Res. 2018;46:D894-D900.

15. Gao F, Al-Azayzih A, Somanath PR. Discrete functions of GSK3alpha and GSK3beta isoforms in prostate tumor growth and micrometastasis. Oncotarget. 2015;6:5947-62.

16. Gao F, Artham S, Sabbineni H, Al-Azayzih A, Peng XD, Hay N, et al. Akt1 promotes stimuli-induced endothelial-barrier protection through FoxO-mediated tight-junction protein turnover. Cellular and molecular life sciences : CMLS. 2016. 
17. Lossi L, Castagna C, Merighi A. Caspase-3 Mediated Cell Death in the Normal Development of the Mammalian Cerebellum. International journal of molecular sciences. 2018;19.

18. Amatschek S, Lucas R, Eger A, Pflueger M, Hundsberger H, Knoll C, et al. CXCL9 induces chemotaxis, chemorepulsion and endothelial barrier disruption through CXCR3-mediated activation of melanoma cells. Br J Cancer. 2011;104:469-79.

19. Taki M, Abiko K, Ukita M, Murakami R, Yamanoi K, Yamaguchi K, et al. Tumor Immune Microenvironment during Epithelial-Mesenchymal Transition. Clinical cancer research : an official journal of the American Association for Cancer Research. 2021;27:4669-79.

20. Ma J, Waxman DJ. Dominant effect of antiangiogenesis in combination therapy involving cyclophosphamide and axitinib. Clinical cancer research : an official journal of the American Association for Cancer Research. 2009;15:578-88.

21. Yang Z, Sun Q, Guo J, Wang S, Song G, Liu W, et al. GRSF1-mediated MIR-G-1 promotes malignant behavior and nuclear autophagy by directly upregulating TMED 5 and LMNB1 in cervical cancer cells. Autophagy. 2019;15:668-85.

22. Damaghi M, West J, Robertson-Tessi M, Xu L, Ferrall-Fairbanks MC, Stewart PA, et al. The harsh microenvironment in early breast cancer selects for a Warburg phenotype. Proc Natl Acad Sci U S A. $2021 ; 118$.

23. Fu Y, Li J, Lee AS. GRP78/BiP inhibits endoplasmic reticulum BIK and protects human breast cancer cells against estrogen starvation-induced apoptosis. Cancer research. 2007;67:3734-40.

24. Tomas-Hernandez S, Blanco J, Rojas C, Roca-Martinez J, Ojeda-Montes MJ, Beltran-Debon R, et al. Resveratrol Potently Counteracts Quercetin Starvation-Induced Autophagy and Sensitizes HepG2 Cancer Cells to Apoptosis. Molecular nutrition \& food research. 2018;62.

25. Jaud M, Philippe C, Di Bella D, Tang W, Pyronnet S, Laurell H, et al. Translational Regulations in Response to Endoplasmic Reticulum Stress in Cancers. Cells. 2020;9.

26. Shen M, Zhao X, Zhao L, Shi L, An S, Huang G, et al. Met is involved in TIGAR-regulated metastasis of non-small-cell lung cancer. Molecular cancer. 2018;17:88.

27. Brabletz T. EMT and MET in metastasis: where are the cancer stem cells? Cancer cell. 2012;22:699701.

28. Tsuji T, Ibaragi S, Shima K, Hu MG, Katsurano M, Sasaki A, et al. Epithelial-mesenchymal transition induced by growth suppressor p12CDK2-AP1 promotes tumor cell local invasion but suppresses distant colony growth. Cancer research. 2008;68:10377-86.

29. Ashrafizadeh M, Mirzaei S, Hashemi F, Zarrabi A, Zabolian A, Saleki H, et al. New insight towards development of paclitaxel and docetaxel resistance in cancer cells: EMT as a novel molecular mechanism and therapeutic possibilities. Biomed Pharmacother. 2021;141:111824.

30. Padmanaban V, Krol I, Suhail Y, Szczerba BM, Aceto N, Bader JS, et al. E-cadherin is required for metastasis in multiple models of breast cancer. Nature. 2019;573:439-44.

31. Reichert M, Bakir B, Moreira L, Pitarresi JR, Feldmann K, Simon L, et al. Regulation of Epithelial Plasticity Determines Metastatic Organotropism in Pancreatic Cancer. Developmental cell. 
Figures

\section{Cell number}

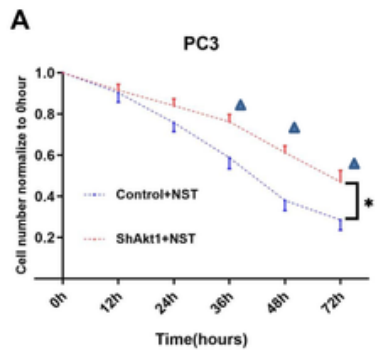

B

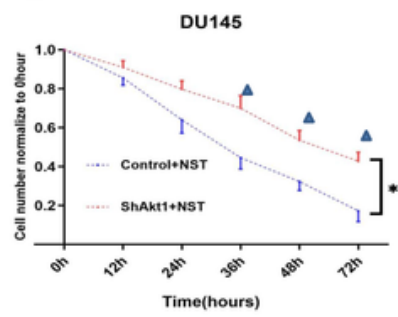

C MTT

D
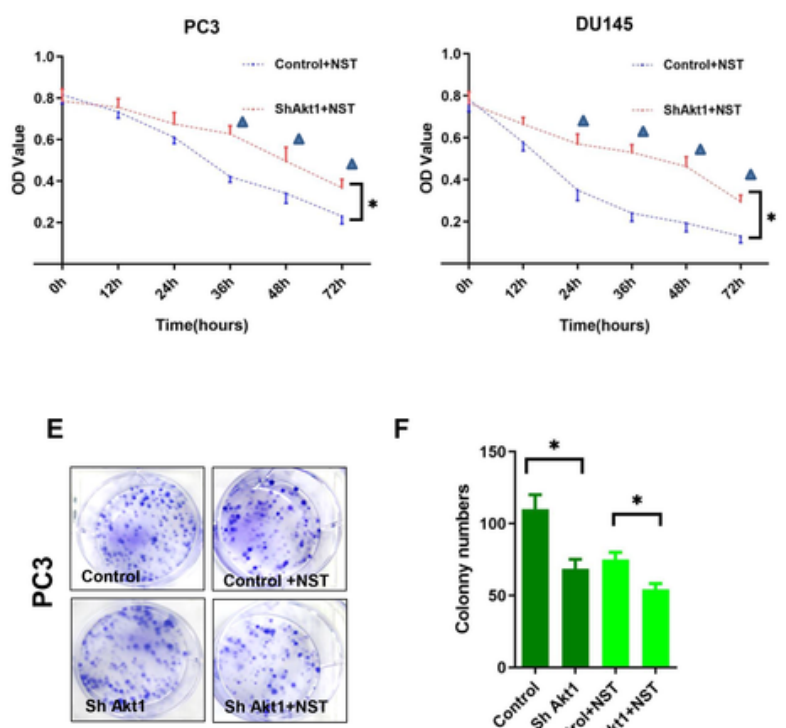

$\mathbf{F}$

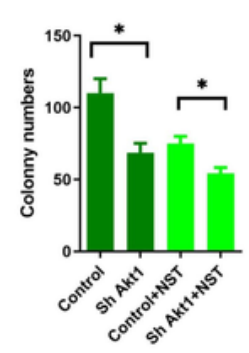

G

H
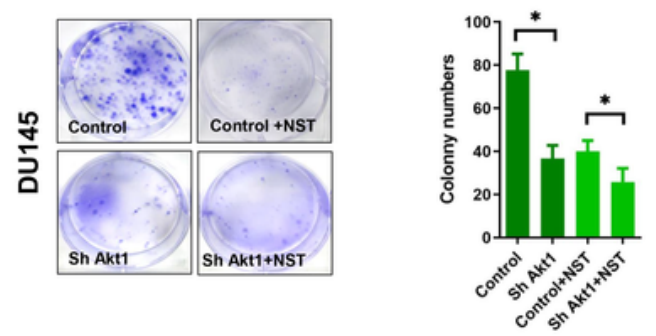

Fig. 1 siliencing Axt1 gene enthanced the resistance of prostate cancer
cells to starvation. A-B, Cell number were revculated in PC 3 and DU 145 colls

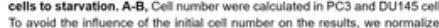
the cell number at each time point to the inhial cell number to get the relative

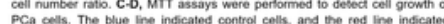
Shater cells. "indicanted pe0.01. Aindicated the comparison botween the two

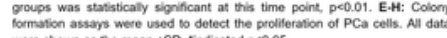
were shom as the mean $\pm S D$, "indicated p<0 05 .

\section{Figure 1}

See image above for figure legend 


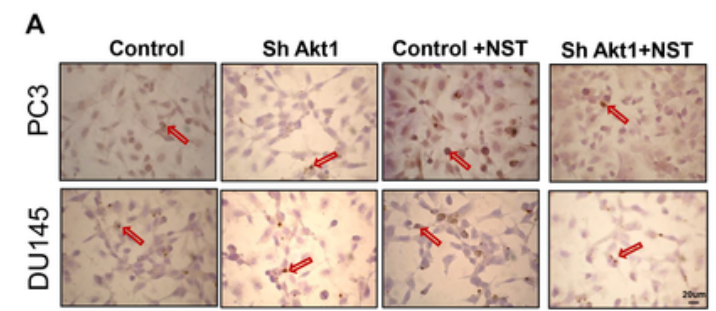

B
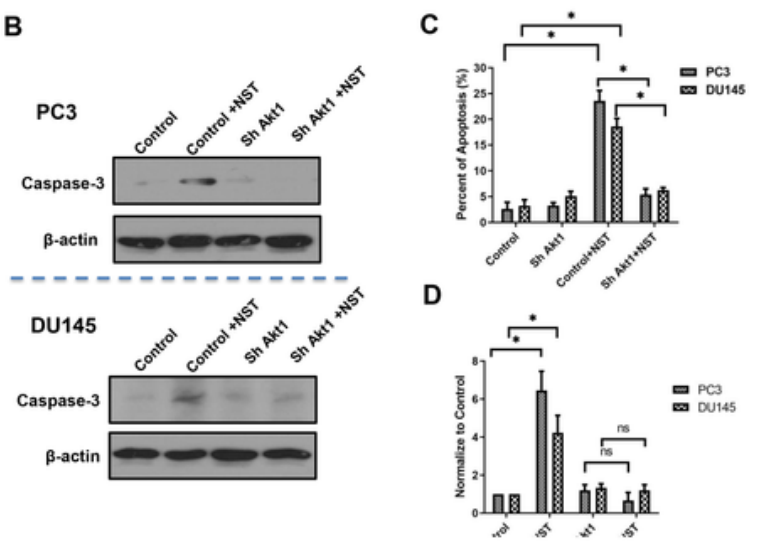

E
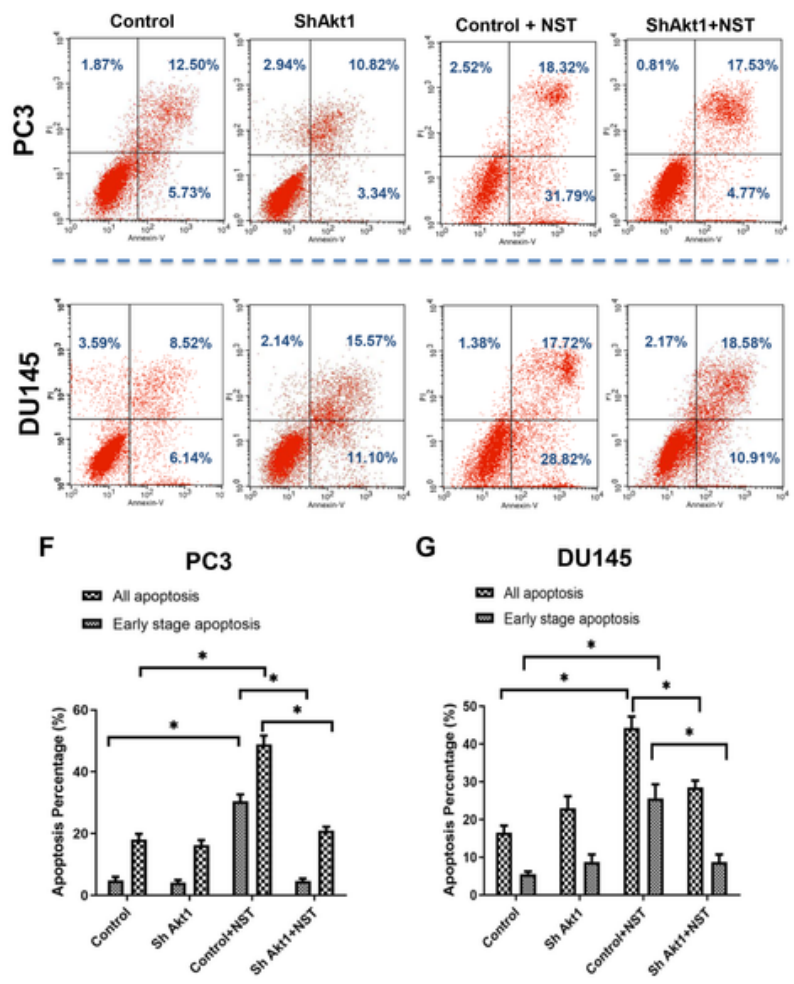

\section{Figure 2}

Silencing Akt1 gene significantly inhibited starvation-induced apoptosis of PCa cells. A, C: Apoptosis cells of PCa were detected by TUNNEL. The apoptosis cells were brown in nuclear. B, D: The western-blot Analysis was performed to detect the expression of Caspase-3 in DU145 and PC3 cells. E-G: Apoptosis cells of PCa were detected by flow cytometry. PI-/Annexin-V+ cells were early-stage apoptosis cells; All 
apoptosis cells were $\mathrm{PI}-/$ Annexin- $\mathrm{V}+$ cells and $\mathrm{PI}+/$ Annexin- $\mathrm{V}+$ cells. Data were shown as the mean $\pm \mathrm{SD}$, * indicated $p<0.01$, ns indicated $p>0.05$. Red arrow indicated the apoptosis cells detected by TUNNEL.
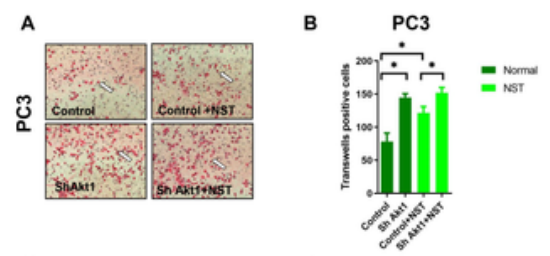

c

D
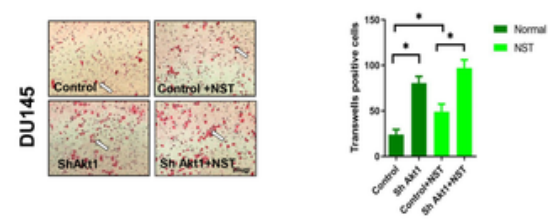

E

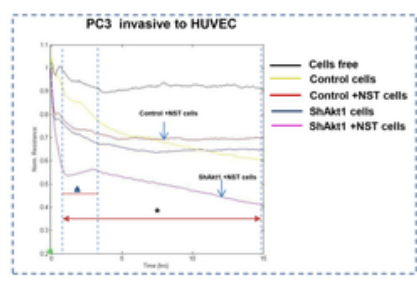

$\mathbf{F}$

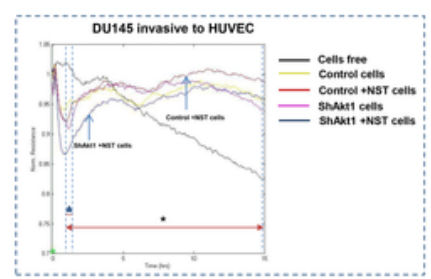

G

Control Sh Akt1 Control +NST Sh Akt1+NST
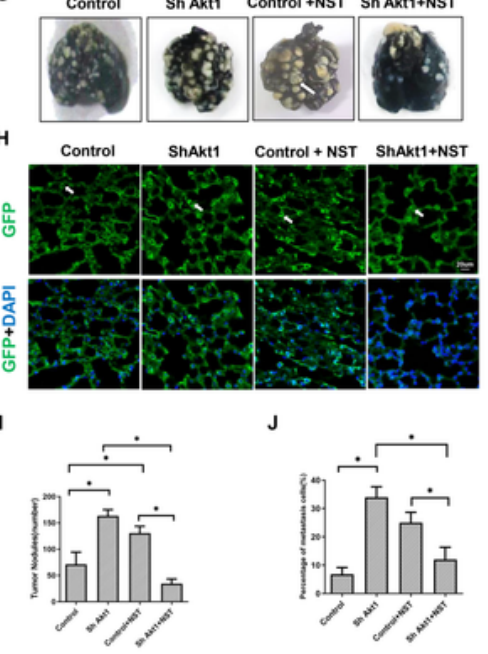

Figure 3

Silencing the Akt1 gene enhanced PCa cell invasion induced by starvation in vitro, however, inhibited lung metastasis induced by starvation in vivo. A-D: The transwells assays were performed to detect the invasion ability of PCa cells. * indicated p<0.01. E-F: The invasion of PCa cells was detected by ECIS. 
*indicated during the time range, there was a significant difference between ShAkt1+NST group and Control +NST group, $p<0.01$; blue triangle indicated during the time range, there was a significant difference between Control group and ShAkt1 group, $p<0.01$. G, I: The lung metastasis of nude mice after two weeks tail vein injection of DU145 cells was detected by India-ink. The tumor nodules were the white dots, and the black tissues are normal lung tissues. * indicated $\mathrm{p}<0.01 . \mathrm{H}, \mathrm{J}$ : The lung metastasis of nude mice after 3 days tail vein injection of DU145 cells detected by ICC. The metastasis PCa cells are GFP positive cells, green in the photos (white arrow indicated metastasis cells). We counted these GFP positive cells and calculated the percentage of metastasis cells (GFP positive cells/total cells). * indicated $p<0.01$ between the two groups $\varangle n=5$. 


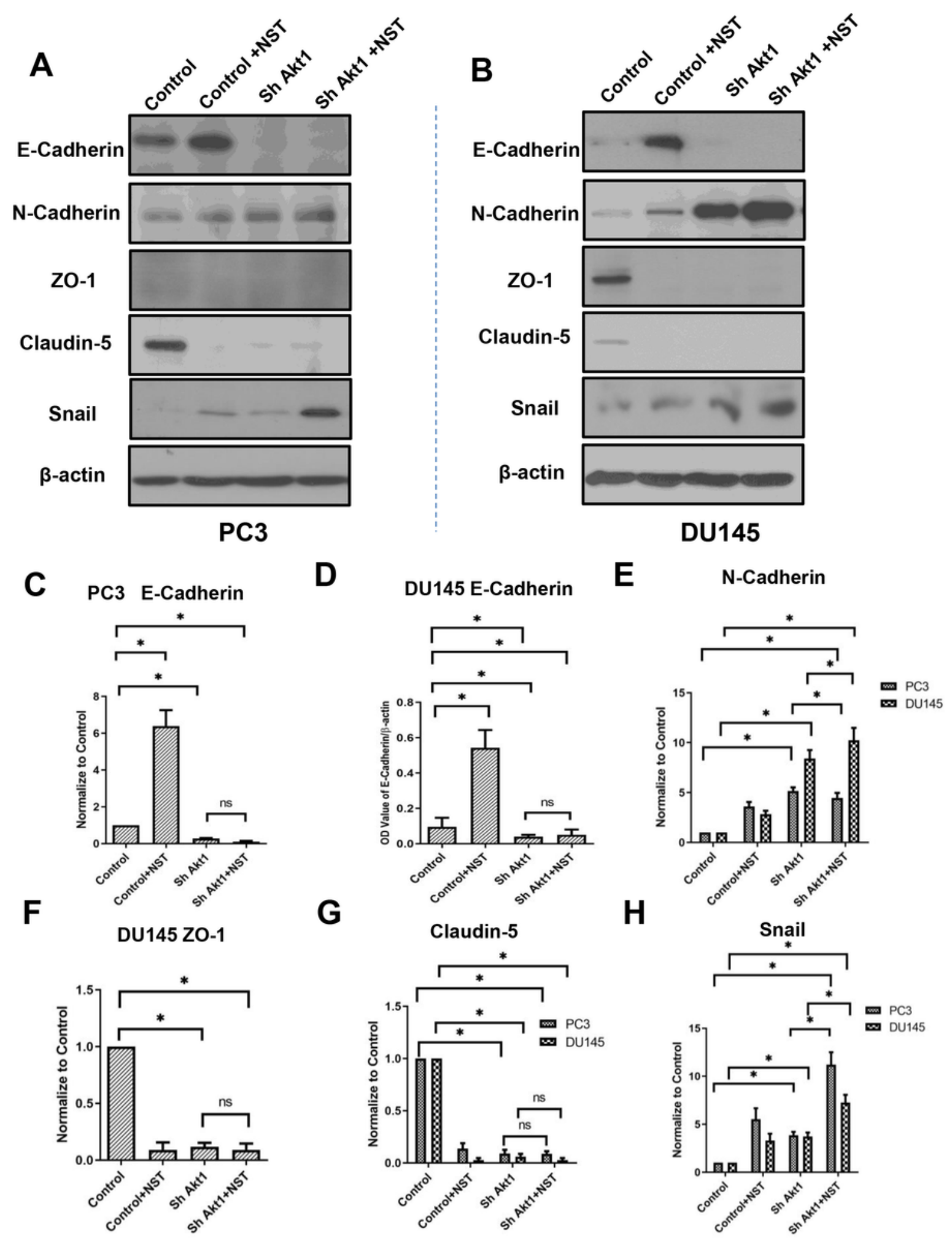

Figure 4

The combination of NST and silencing Akt1 maintained the mesenchymal state of PCa cells. A-B: Western blot assays were performed to detect EMT markers of C3 and DU145 cells. Fig.4A is for PC3, and Fig.4B is for DU145.C-H: The analyzed results of WB in PC3 and DU145 cells. Most WB results were normalized to control. However, for deficient levels in the control group, such as the E-cadherin level of 
DU145, we use the ratio between the target protein and $\beta$-actin. The ZO- 1 levels of PC3 were shallow in each group, so we did not show its analyzed results. *indicated $p<0.01$, ns indicated $p>0.05, n=3$.

\section{Supplementary Files}

This is a list of supplementary files associated with this preprint. Click to download.

- supplementaryFigures.pptx

- SupplementaryFigureLegends.docx

- supplementarytable.pptx 